\title{
Imaging Individual Molecular-Like Orbitals of a Non-Planar Naphthalene Diimide on Pt(111): A Combined STM and DFT Study
}

\author{
R. Ebeling, ${ }^{\dagger}$ S. Tsukamoto, ${ }^{\ddagger}$ E. Dirksen, ${ }^{\S}$ V. Caciuc, ${ }^{\ddagger}$ T. J. J. Müller, ${ }^{\S}$ N. Atodiresei, ${ }^{*}$, \\ and S. Karthäuser* ${ }^{*} \dagger$ \\ ${ }^{\dagger}$ Peter Grünberg Institut (PGI-7) and JARA-FIT, Forschungszentrum Jülich GmbH, Jülich 52425, Germany \\ ${ }^{*}$ Peter Grünberg Institut (PGI-1) and Institute of Advanced Simulations (IAS-1), Forschungszentrum Jülich GmbH and JARA, Jülich \\ 52425, Germany \\ ${ }^{\S}$ Institut für Organische Chemie und Makromolekulare Chemie, Heinrich-Heine-Universität Düsseldorf, Universitätsstrasse 1, \\ Düsseldorf 40225, Germany
}

Supporting Information

ABSTRACT: Functionalized naphthalene diimides (NDIs) are attractive candidates as small molecule acceptors for various molecular electronic applications due to their reversible two-step one-electron reductions at relatively low potentials. Here, we utilize low-temperature scanning tunneling microscopy (STM) to study the spatial extent and the electronic structure of 2,7-dibenzyl 1,4,5,8-ñaphthalenetetraçarboxylic diimide (BNTCDI) adsorbed on the $\mathrm{Pt}(111)$ surface. We succeeded to map in real space the electronic structure of this three-dimensional (3D) molecule with orbital resolution, and thus were able to image an in-plane $\pi$-nodal plane located at the benzyl side arms. Furthermore, on the basis of
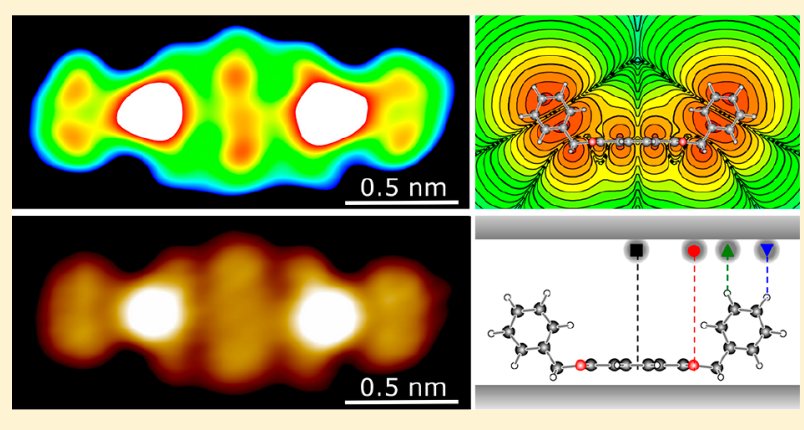
density functional theory simulations, we are able to explain the STM features of BNTCDI in terms of a convolution between its 3D shape and electronic structure. Importantly, for this weakly coupled molecule on the $\mathrm{Pt}(111)$ substrate, the intramolecular $\mathrm{N} \cdots \mathrm{H}-\mathrm{C}$ hydrogen bonds (i) stabilize the protruding $\pi$-systems of the benzyl groups perpendicular to the flat NDI core and (ii) open an effective transport path around Fermi energy.

\section{INTRODUCTION}

Naphthalene diimides (NDIs) have received considerable interest in recent years as functional small molecule acceptors ${ }^{1,2}$ for various molecule based electronics applications, such as nonfullerene acceptors in organic photovoltaics, ${ }^{3-6}$ as n-type semiconductors, ${ }^{7-9}$ in donor-acceptor conjugates, ${ }^{10-12}$ and in electrochemical sensors. ${ }^{13,14}$ Particularly interesting are their Nernstian reversible two-step one-electron reductions at relatively low potentials, which also can be adjusted by core substitutions in a broad range. ${ }^{15}$ With this regard, LUMO energies of $3.7 \mathrm{eV}$ can be readily achieved. In addition, the ease of preparation of symmetrically and unsymmetrically substituted NDIs by imidation of commercially available 1,4,5,8naphthalenetetracarboxylic dianhydride by primary amines is very attractive for accessing functionalized NDI with fine-tuned properties.

In order to investigate molecular orbital structures and transport properties in detail, low-temperature ultrahigh vacuum scanning tunneling microscopy (LT-UHV-STM) is a powerful technique that provides real space images of surface structures with local atomic resolution and, thus, triggered seminal developments in surface science. Using this technique, the tunneling current between a sharp metal tip, placed in close proximity to a sample $(<1 \mathrm{~nm})$, and the sample surface is measured while a small bias voltage is applied and the tip is scanning the surface. Thereby, the local electron density of states (LDOS) between the tip and a conducting substrate is detected. If a molecule is placed between the tip and the substrate, the modulation of the LDOS by the molecule is recorded. ${ }^{16-19}$ In the case of planar, weakly coupled molecules, such as pentacene and other aromatic hydrocarbons, highresolution images of the molecular orbital structure can be obtained, especially if a very thin electronic decoupling layer of $\mathrm{NaCl}$ is placed between the metallic substrate and the molecule. ${ }^{20}$ Applying this method, it is possible to image the frontier orbitals of molecules on substrates that resemble the molecular orbitals of the free molecule in the gas phase. Besides $\mathrm{NaCl}$, other materials, such as graphene, hexagonal boron nitride, transition metal dichalcogenides, or oxides, may serve as an electronic decoupling layer enabling the direct imaging of molecular orbitals of planar molecules. ${ }^{21-26}$ However, if a $\pi$ conjugated planar molecule is chemisorbed onto a metal

Received: October 6, 2017

Revised: November 14, 2017

Published: November 14, 2017 
Scheme 1. Synthesis of BNTCDI

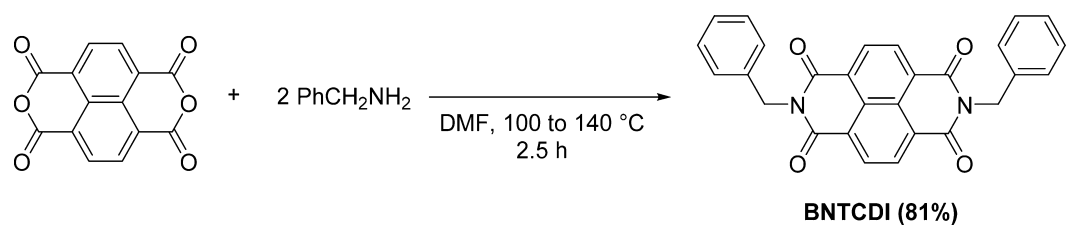

substrate, it is difficult to obtain molecular orbital resolution with STM due to the direct coupling of molecular states with the electronic states of the metal. ${ }^{27}$ Access to the geometrical structure of planar molecules is obtained by scanning tunneling hydrogen microscopy ${ }^{28}$ or tuning-fork-based noncontact atomic force microscopy. ${ }^{27,29-31}$ Thus, molecular structure, bond order, and even intermolecular features in molecular networks could be characterized. If, in addition, electronic molecular properties should be investigated, a combined STM and NC-AFM study is useful for two-dimensional (2D) molecules.

However, the detailed STM characterization of the molecular conformation and the electronic structure of a three-dimensional (3D) molecule adsorbed on a metallic surface is very challenging. Generally speaking, the STM images are a rather complicated convolution of the sample geometry and the probed electronic structure. As compared to $2 \mathrm{D}$ molecular structures, in the case of $3 \mathrm{D}$ molecules, this situation gets even more complicated, since the tunneling current scales exponentially with the tip-sample distance, and thus, height differences within a molecule cause considerable variations of the current signal and, thereby, lead to a decreased resolution. Consequently, it is even more important for a $3 \mathrm{D}$ molecule to be electronically decoupled from the metallic surface in order to enable orbital resolution. However, if a functional molecule should be characterized in device geometry or at least in contact with the same metal surface as in a device, another approach has to be adopted. Recently, the introduction of spatially demanding substituents has been employed to decouple molecules from a metal surface. ${ }^{32,33}$ These substituents induce a steric hindrance, leading to a measurable increase in the molecule-substrate distance and, thus, to an electronic decoupling. This method bears the advantage that the degree of decoupling can be steered by the size of the substituent.

Here, we apply a minor steric decoupling to study the hybrid interface formed by 2,7-dibenzyl 1,4,5,8-ñaphthalenetetracarboxylic diimide (BNTCDI) adsorbed on $\mathrm{Pt}(111)$. More precisely, the anchoring part of our molecule, the NDI backbone, contains four oxygen atoms known to weakly chemisorb a molecular planar structure on metallic surfaces. ${ }^{32,34,35}$ Therefore, two kinks represented by the methylene groups of the benzyl side arms are introduced at both sides of the NDI core to provide a steric hindrance so that the molecule is slightly lifted from the surface. As a consequence, the benzyl side arms of BNTCDI are expected to protrude from the plane of the molecular core, causing a non-planarity of the molecule. ${ }^{9,11}$ In this study, we provide a detailed insight into the adsorption geometry and electronic structure of this 3D molecule adsorbed on $\mathrm{Pt}(111)$, as revealed by LT-UHV-STM and density functional theory (DFT) simulations.

\section{EXPERIMENTAL METHODS}

Chemical Synthesis and Analysis. 1,4,5,8-Naphthalenetetracarboxylic dianhydride $(1.34 \mathrm{~g}, 5.00 \mathrm{mmol})$ and benzylamine $(1.17 \mathrm{~g}, 10.9 \mathrm{mmol})$ were dissolved in DMF $(20 \mathrm{~mL})$ and stirred at RT for $2 \mathrm{~h}$ (Scheme 1$).^{36}$ The reaction mixture was then stirred at $100{ }^{\circ} \mathrm{C}$ for $2 \mathrm{~h}$ and at $140{ }^{\circ} \mathrm{C}$ for another 30 $\min$. The resulting suspension was poured into cold water, and the precipitate was filtered and dried under vacuum. Purification was performed by recrystallization from dichloromethane to give 2,7-dibenzyl 1,4,5,8-naphthalenetetracarboxylic diimide as a cream-colored solid (1.82 g, 81\%), $\mathrm{Mp}>257^{\circ} \mathrm{C}$ (dec).

${ }^{1} \mathrm{H}$ NMR $\left(300 \mathrm{MHz} \mathrm{CDCl}_{3}\right): \delta 5.39(\mathrm{~s}, 4 \mathrm{H}), 7.23-7.35(\mathrm{~m}$, $6 \mathrm{H}), 7.52-7.56(\mathrm{~m}, 4 \mathrm{H}), 8.76(\mathrm{~s}, 4 \mathrm{H}) .{ }^{13} \mathrm{C} \mathrm{NMR}(75 \mathrm{MHz}$, $\left.\mathrm{CDCl}_{3}\right): \delta 44.1\left(\mathrm{CH}_{2}\right), 126.8\left(\mathrm{C}_{\text {quat }}\right), 128.0(\mathrm{CH}), 128.7(\mathrm{CH})$, $129.2(\mathrm{CH}), 131.3(\mathrm{CH}), 136.6\left(\mathrm{C}_{\text {quat }}\right), 162.9\left(\mathrm{C}_{\text {quat }}\right)$.

$\mathrm{EI}+\mathrm{MS}(m / z(\%)): 447(30), 446\left([\mathrm{M}]^{+}, 100\right), 341(11)$ $340\left(\left[\mathrm{M}-\mathrm{C}_{7} \mathrm{H}_{7} \mathrm{~N}\right]^{+}, 42\right), 312(30), 91(22)$.

FT-IR: $\tilde{\nu}\left[\mathrm{cm}^{-1}\right]=630(\mathrm{~s}), 669(\mathrm{~s}), 711(\mathrm{~m}), 737(\mathrm{w}), 745$ (m), $770(\mathrm{~s}), 810(\mathrm{w}), 881(\mathrm{w}), 899(\mathrm{w}), 918(\mathrm{w}), 930(\mathrm{w})$, $957(\mathrm{w}), 970(\mathrm{w}), 984(\mathrm{w}), 995(\mathrm{~m}), 1028(\mathrm{w}), 1074(\mathrm{~m})$, $1103(\mathrm{w}), 1142(\mathrm{w}), 1180(\mathrm{~m}), 1217(\mathrm{w}), 1242(\mathrm{~s}), 1288(\mathrm{w})$, $1302(\mathrm{~m}), 1333$ (s), $1368(\mathrm{~m}), 1393(\mathrm{w}), 1418(\mathrm{w}), 1450(\mathrm{~m})$, $1495(\mathrm{w}), 1558(\mathrm{w}), 1580(\mathrm{~m}), 1607(\mathrm{w}), 1649(\mathrm{~m}), 1661(\mathrm{~s})$, $1703(\mathrm{~m})$.

Anal. Calcd for $\mathrm{C}_{28} \mathrm{H}_{18} \mathrm{~N}_{2} \mathrm{O}_{4}$ (446.5): C 75.33, $\mathrm{H}$ 4.06, $\mathrm{N}$ 6.27; Found: C 75.17, H 4.12, N 6.19.

$\mathrm{UV} / \mathrm{vis}\left(\lambda_{\max }[\mathrm{nm}]\left(\varepsilon\left[\mathrm{L} \mathrm{mol}{ }^{-1} \mathrm{~cm}^{-1}\right]\right)\right): 343$ (13000), 361 (20200), 381 (27700).

The cyclic voltammogram was recorded in $\mathrm{CH}_{2} \mathrm{Cl}_{2}$ at $293 \mathrm{~K}$ with a $\mathrm{Pt}$ working electrode, $\mathrm{Pt}$ counter electrode, $\mathrm{Ag} / \mathrm{AgCl}$ reference electrode, $v=100 \mathrm{mV} / \mathrm{s}$, standard decamethyl ferrocene, electrolyte $0.1 \mathrm{M}\left[\mathrm{Bu}_{4} \mathrm{~N}\right]\left[\mathrm{PF}_{6}\right] . E_{1 / 2}{ }^{0 /-1}=-0.580 \mathrm{~V}$, and $E_{1 / 2}{ }^{-1 /-2}=-0.998 \mathrm{~V}$.

Scanning Tunneling Microscopy Details. A commercial Createc STM (Germany) was used to perform all lowtemperature scanning tunneling microscopy (LT-STM) and spectroscopy (STS) experiments under ultrahigh vacuum (UHV) with a base pressure of $1 \times 10^{-10} \mathrm{mbar}$. The UHVLT-STM is composed of three parts, i.e., a load-lock, a preparation chamber equipped with LEED, and a measurement chamber. The system is operated at 4.2 or $77 \mathrm{~K}$ using custommade electrochemically etched tungsten tips. All STM images were recorded in constant-current mode with the bias voltage applied to the sample. The data are plotted after applying plane correction and optional noise filtering by a slight Fourier filter using SPIP. Molecular heights given in images correspond to averaged apparent heights obtained from height profiles. The given lengths correspond to the full width half maxima of the structures. Differential conductance $(\mathrm{d} I / \mathrm{d} V)$ spectra were recorded after switching-off the feedback loop using lock-in detection to record the tunneling current while the bias voltage was modulated. The spectroscopic data were obtained on several molecules spread over the samples and usually averaged 
over five curves. The measurement parameters are given directly in the respective captions of the STM/STS measurements.

The substrate used in STM measurements was a polished Pt(111) single crystal (purity 99.999\%), which was purchased from MaTeck (Germany). It was cleaned by several cycles of $\mathrm{Ne}^{+}$-ion sputtering $(1 \mathrm{kV}, 30 \mathrm{~min}, 2.5 \mu \mathrm{A})$, baking $\left(600^{\circ} \mathrm{C}, 1 \times\right.$ $10^{-6}$ mbar of $\mathrm{O}_{2}$ partial pressure), and subsequent thermal annealing $\left(900{ }^{\circ} \mathrm{C}, 20 \mathrm{~min}\right)$. The cleanliness of the $\mathrm{Pt}(111)$ surface was checked by STM inspection and LEED measurements. The cleaning procedure was pursued until large clean terraces with a diameter up to several $100 \mathrm{~nm}$ in STM and sharp spots with a hexagonal symmetry in LEED appeared.

BNTCDI molecules were outgassed and subsequently deposited onto the clean $\operatorname{Pt}(111)$ crystal by sublimation at $120-150{ }^{\circ} \mathrm{C}$ using a Knudsen cell. During the submonolayer deposition, the background pressure was kept in the $10^{-10} \mathrm{mbar}$ range and the substrate surface at room temperature (RT).

Computational Details. The tunneling electron transport calculations are carried out employing the scattering wave function code juTRANS ${ }^{37}$ based on the Lippmann-Schwinger equation formalism. ${ }^{38}$ Within the framework of the density functional theory, in the present simulations, we used the projector-augmented-plane wave $\operatorname{method}^{39}$ and the local density approximation ${ }^{40}$ for the exchange-correlation potential. The electron transmission spectra were taken by means of the Landauer-Büttiker formulism. $^{41}$

\section{RESULTS AND DISCUSSION}

Synthesis and Electrochemical Characterization. The NDI derivative BNTCDI was prepared by condensation of 1,4,5,8-naphthalenetetracarboxylic dianhydride with benzyl amine in DMF at 100 and $140{ }^{\circ} \mathrm{C}$ in $81 \%$ yield, as shown in Scheme $1 .{ }^{36}$ The molecular structure was unambiguously assigned by ${ }^{1} \mathrm{H}$ and ${ }^{13} \mathrm{C}$ NMR spectroscopy and mass spectrometry, and the elemental composition was confirmed by combustion analysis.

Furthermore, thermogravimetric (TG) and differential thermal (DT) analysis were performed to elucidate the thermal behavior of BNTCDI in a temperature range from room temperature to $650{ }^{\circ} \mathrm{C}$ (see Figure S1 in the Supporting Information). Preceding a sharp melting point at $271^{\circ} \mathrm{C}$, a minor endothermic peak indicates a phase change. BNTCDI is thermally stable up to $300{ }^{\circ} \mathrm{C}$, where an endothermic decomposition begins as indicated by a steady mass loss.

The UV/vis absorption of BNTCDI was determined in dichloromethane solutions showing three absorption maxima at 343,361 , and $381 \mathrm{~nm}$ with molar extinction coefficients of 13000,20200 , and $27700 \mathrm{~L} \mathrm{~mol}^{-1} \mathrm{~cm}^{-1}$, respectively (Figure S2 in the Supporting Information). Cyclic voltammetry gives the first and second Nernstian reversible one-electron reductions at $E_{1 / 2}{ }^{0 /-1}=-0.58 \mathrm{~V}$ and $E_{1 / 2}{ }^{-1 /-2}=-1.00 \mathrm{~V}$ as referenced to the NHE (normal hydrogen electrode, Figure S3 in the Supporting Information). These values are in good agreement with literature data of $\mathrm{N}, \mathrm{N}$-dialkyl NDIs. ${ }^{15}$

Adsorption Geometry and Electronic Structure of BNTCDI. BNTCDI can be sublimed under ultrahigh vacuum conditions from powder samples onto $\mathrm{Pt}(111)$ substrates in a highly reproducible manner concerning the resulting molecule coverage. LT-UHV-STM investigations reveal elongated features with an apparent height of 90-120 pm, which can be assigned to single BNTCDI molecules (Figure 1). Besides BNTCDI, some adsorbates characteristic for $\mathrm{Pt}(111)$ with an

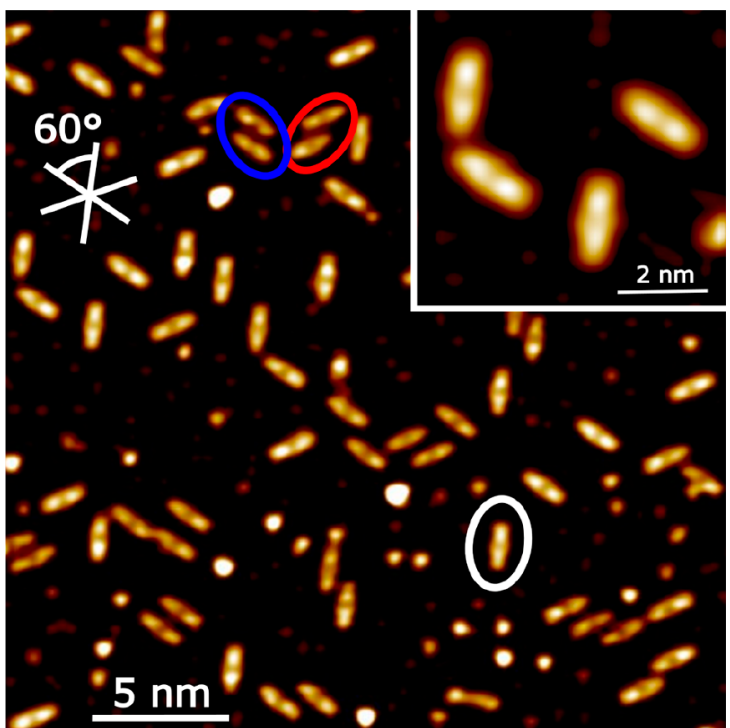

Figure 1. STM image showing BNTCDI (white encircled), dimers (blue and red encircled, see text), and some molecular fragments $\left(U_{\text {bias }}\right.$ $=0.1 \mathrm{~V}, I_{\text {set }}=200 \mathrm{pA}, T=4.2 \mathrm{~K}$ ). The three directions adopted by the elongated molecules are indicated by white lines. The inset shows a close-up of the molecules.

apparent height up to $100 \mathrm{pm}$, like residual oxygen or hydrogen atoms, and a few molecular fragments are observable. A molecular length of $1.9 \mathrm{~nm}$ and a width of $0.7 \mathrm{~nm}$ were obtained from the STM images and are in accordance with theoretically deduced molecule dimensions. BNTCDI on $\mathrm{Pt}(111)$ adsorbs along three directions spanning an angle of 60 or $120^{\circ}$ with respect to each other. Hence, there is a 3 -fold degenerated bonding site of BNTCDI on the hexagonal $\mathrm{Pt}(111)$ surface and commensurability can be assumed.

Furthermore, dimer formation can be observed, even though a submonolayer deposition was aspired in order to receive isolated molecules on the surface (Figure 1). It is known for unsubstituted and monosubstituted NDI compounds that they are able to self-assemble through $\mathrm{N}-\mathrm{H} \cdots \mathrm{O}=\mathrm{C}$ and $\mathrm{C}-\mathrm{H} \cdots$ $\mathrm{O}=\mathrm{C}$ hydrogen bonds generating supramolecular networks. ${ }^{30,42-44}$ In the case of disubstituted NDI compounds, like BNTCDI, only the weak intermolecular $\mathrm{C}-\mathrm{H} \cdots \mathrm{O}=\mathrm{C}$ hydrogen bonds are available. However, these might lead to dimers or, at higher coverage, brickwork structures like those reported for dialkyl substituted NDI derivatives. ${ }^{45,46}$ BNTCDI dimers adsorbed on a surface may adopt at least two intermolecular geometries defined by the displacements along the long and short axes within the NDI plane that allow reduction of the $\mathrm{C}-\mathrm{H} \cdots \mathrm{H}-\mathrm{C}$ repulsion and the formation of two $\mathrm{C}-\mathrm{H} \cdots \mathrm{O}=\mathrm{C}$ bonds (blue and red encircled dimers in Figure 1). These intermolecular interactions, the moleculesurface interactions, and the low coverage are responsible for the formation of the different dimer geometries observed here.

The high-resolution STM image of a single BNTCDI molecule on $\mathrm{Pt}(111)$ (Figure 2) reveals that the molecules exhibit $C_{2 v}$ symmetry, i.e., show two mirror planes, which intersect the molecule center and are perpendicular to each other. All molecules without exception (see Figure 1) exhibit this appearance, pointing to a local energy minimum of this conformation. This molecular appearance can be explained straightforwardly by the adsorption of the NDI part flat on the $\mathrm{Pt}(111)$ substrate, while the phenyl groups on both sides stand 
(a)
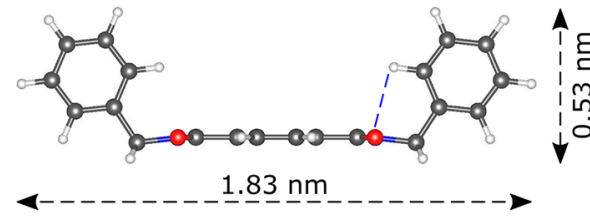

(b)

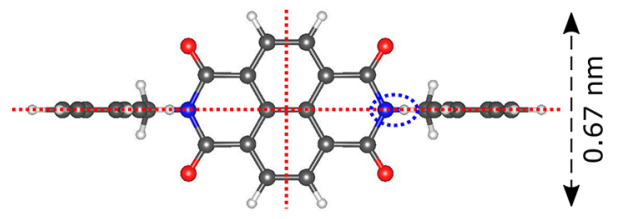

(c)

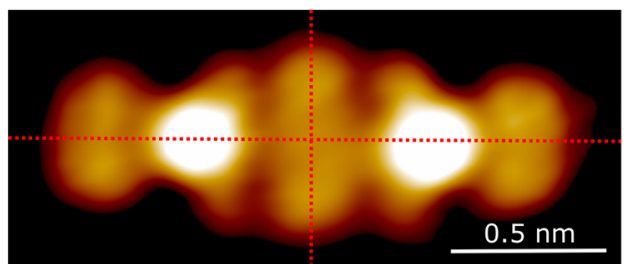

(d)

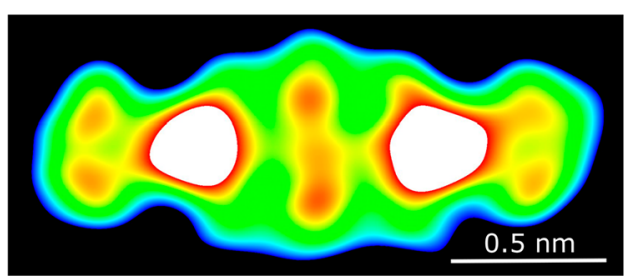

Figure 2. Sketch of BNTCDI: (a) side view; (b) top view with indicated mirror planes. One hydrogen bond between an $\mathrm{N}$ atom of the naphthoic diimide core and the next $\mathrm{H}$ atom of the phenyl ring $(\mathrm{N} \cdots \mathrm{H}$ distance $0.262 \mathrm{~nm})$ is indicated by a dashed blue line in the side view (a) and an ellipsoid in the top view (b). (c) High resolution UHV-STM image of a single BNTCDI molecule; the mirror planes are indicated $\left(U_{\text {bias }}=+0.5 \mathrm{~V}, I_{\text {set }}=510 \mathrm{pA}, T=4.2 \mathrm{~K}\right)$. The unfiltered STM image and the apparent height profile are given in Figure S4 in the Supporting Information. (d) Contrast enhanced illustration of BNTCDI.

upright, as shown in the schemes given in Figure 2a,b. If the phenyl groups would adsorb flat on the substrate, as suggested by the molecular chemical structure depicted in Scheme 1, the STM images of the BNTCDI would have an unsymmetrical appearance. In addition, we can exclude a fast transformation from one unsymmetrical conformation into the corresponding opposite unsymmetrical conformation by rotation of benzyl groups, resulting in a timely weighted average and, thus, in a symmetrical conformation. Rotations of molecular groups, such as benzyl or phenyl groups, require activation energies in the range from 100 to $400 \mathrm{meV}^{47,48}$ and are frozen at $4.2 \mathrm{~K}$. Consequently, the STM images reflect the molecular orbital structure of BNTCDI with phenyl rings standing upright at both sides, resulting in a three-dimensional molecule.

The observed upright configuration of the phenyl groups in BNTCDI is unaffected by the direction of the STM tip scanning across the molecule or varying set points in the range of $-2<U_{\text {bias }} / \mathrm{V}<2$. We attribute this conformational stability to the introduced methylene linker between the NDI part and the phenyl groups in addition to $\mathrm{N} \cdots \mathrm{H}-\mathrm{C}$ hydrogen bonds. By performing theoretical calculations on BNTCDI in the gas phase, we could identify a close distance of only $0.262 \mathrm{~nm}$ between the nitrogens of the NDI core and hydrogen atoms located at the phenyl rings on both sides of the molecule resembling weak $\mathrm{N} \cdots \mathrm{H}-\mathrm{C}$ hydrogen bonds (see Figure 2a indicated by a blue line). The $\mathrm{N} \cdots \mathrm{H}$ distance is smaller than the sum of the van der Waals radii and smaller than the distance found for the hydrogen bond between pyridine and methyl fluoride which amounts to $0.267 \mathrm{~nm} .{ }^{49,50}$ Consequently, the presence of the $\mathrm{N} \cdots \mathrm{H}$ hydrogen bonds determines (i) the upright configuration of the phenyl groups and (ii) the orientation of the methylene groups with their hydrogen atoms pointing toward the substrate, leading to a considerable decoupling of the phenyl groups from the metal as well as from the NDI $\pi$-electron system.

As depicted in Figure 2c, at the molecular site, we identify characteristic topographic features with intramolecular resolution that indicate a weak electronic coupling between BNTCDI and the substrate due to the decoupling mechanism related to the built-in steric hindrance. Surprisingly, although BNTCDI exhibits a significant 3D geometrical corrugation (see Figure 2a,b), a closer look at the LT-UHV-STM image given in Figure $2 \mathrm{c}$ reveals a spatial resolution of the electron density located at the planar upright phenyl arms as well as at the flat lying NDI core resembling molecular orbital features of the free molecule, as will be discussed later in comparison with DFT calculations.

In order to get further insights into the molecular-like orbital structure of the adsorbed BNTCDI, we performed thorough voltage dependent imaging. By convention, at the molecular site, the electronic states corresponding to unoccupied molecular-like orbitals are measured with positive bias voltages, while the occupied molecular-like orbitals are obtained at negative values of the applied bias voltage. Figure 3 depicts representative high resolution UHV-STM images obtained for single BNTCDI molecules on $\mathrm{Pt}(111)$. First, we note that, from a structural point of view, the absolute length and width of (a)

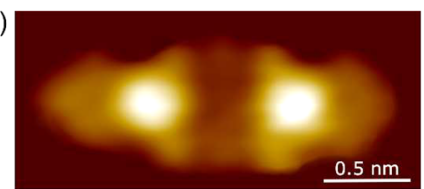

(b)

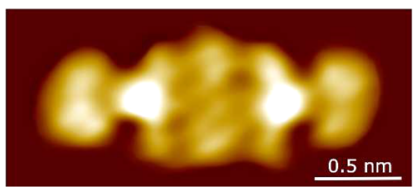

(c)

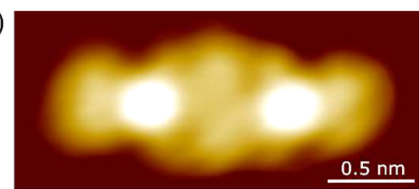

(d)

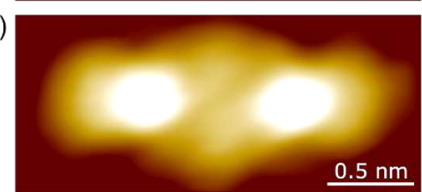

(e)

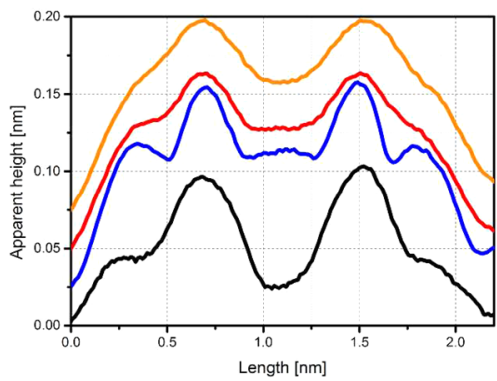

Figure 3. Voltage dependent STM images of BNTCDI on $\operatorname{Pt}(111)$ with submolecular resolution: $(\mathrm{a}) U_{\text {bias }}=-1.5 \mathrm{~V},(\mathrm{~b}) U_{\text {bias }}=+0.25 \mathrm{~V},(\mathrm{c}) U_{\text {bias }}=$ $+1.0 \mathrm{~V},(\mathrm{~d}) U_{\text {bias }}=+2.0 \mathrm{~V}\left(I_{\text {set }}=700 \mathrm{pA}\right)$. (e) Apparent height profiles along the long molecule axis in (a) black, (b) blue, (c) red, and (d) orange (curves shifted by $0.025 \mathrm{~nm}$ for clarity). Raw data together with additional height profiles are given in Figures S5 and S6 in the Supporting Information. 

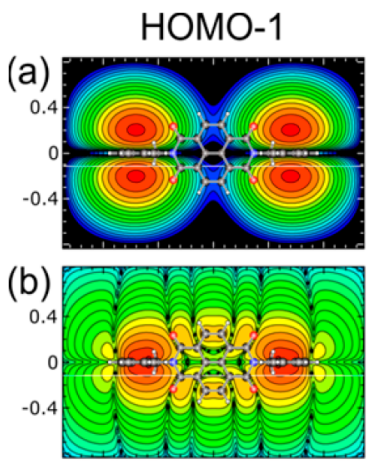

(c)

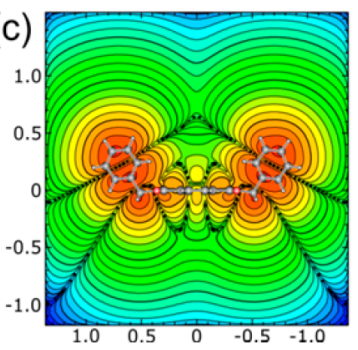

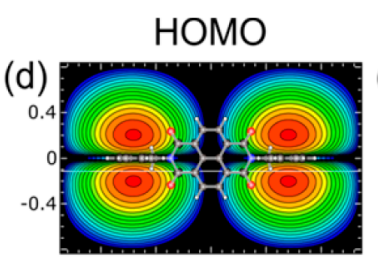
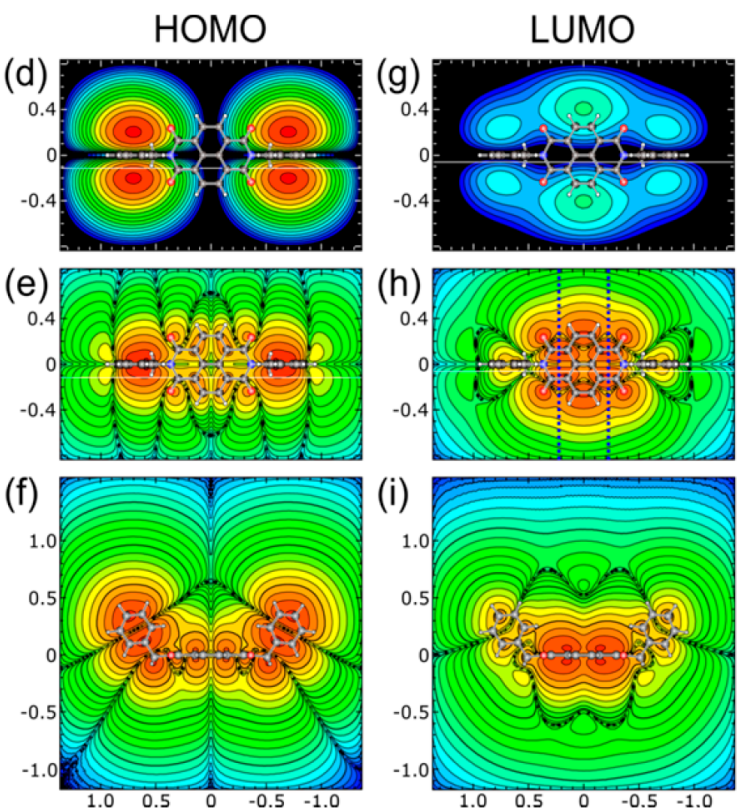

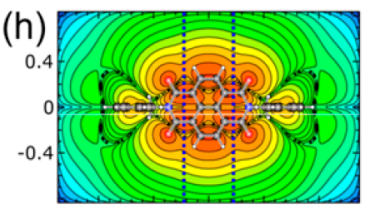

(i)

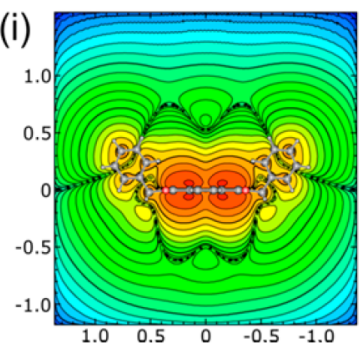

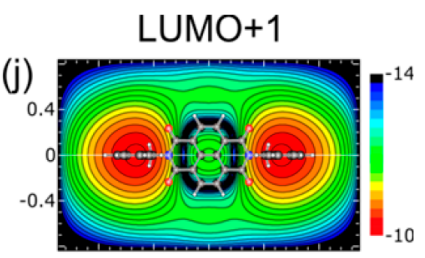
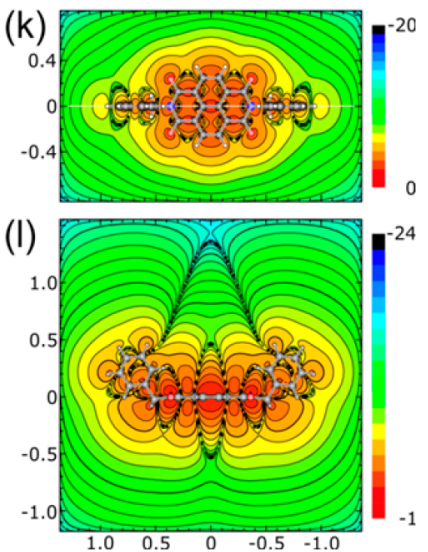

Figure 4. Real space plots (units: $\mathrm{nm}$ ) of the charge density plots on a logarithmic scale (units: e/bohr ${ }^{3}$ ) for the last two occupied (HOMO-1, HOMO) and first two unoccupied (LUMO, LUMO+1) frontier molecular orbitals obtained for the BNTCDI molecule in the gas phase. The top views are cuts through a parallel plane above the naphthoic diimide core at $1 \mathrm{~nm}(\mathrm{a}, \mathrm{d}, \mathrm{g}, \mathrm{j})$ and $0.2 \mathrm{~nm}(\mathrm{~b}, \mathrm{e}, \mathrm{h}, \mathrm{k})$. The side views (c, f, i, l) represent cuts along the white lines indicated in the corresponding top views.

the BNTCDI molecule remain constant at 1.90 and $0.74 \mathrm{~nm}$ with a variance of $\pm 0.03 \mathrm{~nm}$, respectively, independent of the applied bias voltage. In addition, all STM images show two characteristic bright spots, presumably located above the nitrogen atoms of the NDI backbone, with a constant distance of about $0.8 \mathrm{~nm}$. It is also important to note that in all STM images the topographic features are given in a direction perpendicular to the $\mathrm{Pt}(111)$ surface, i.e., in a direction perpendicular to the NDI plane and in a direction parallel to the phenyl planes.

The occupied electronic states recorded at $-1.5 \mathrm{~V}$ are depicted in Figure 3a. They show the highest apparent height difference between the bright spots attributed to $\mathrm{N}$ atoms and the center of the NDI as compared to the STM images obtained at other voltages (Figure $3 \mathrm{e}$ ). In addition, the phenyl rings at the periphery of the BNTCDI appear clearly higher than the molecular center (e.g., the NDI core) and show a tapered shape with a total width of $0.48 \mathrm{~nm}$ at this voltage.

At a bias voltage of $0.25 \mathrm{~V}$, a considerably different appearance of the molecular-like orbitals can be observed, as given in Figure $3 \mathrm{~b}$. The characteristic bright spots located above the $\mathrm{N}$ atoms are less dominant at this bias voltage as compared to those imaged at $-1.5 \mathrm{~V}$, allowing the identification of other subtle features indicating a submolecular resolution. One such remarkable feature is the high electron density at the periphery of the NDI backbone compared to its center. Moreover, in a direction perpendicular to the phenyl arms, a depression of 7 $\mathrm{pm}$ at the phenyl atomic plane can be determined. Note that such an intramolecular resolution is similar to that observed for physisorbed molecules, ${ }^{20,25}$ suggesting a weak molecule/ $\mathrm{Pt}(111)$ interaction. On the other hand, such submolecular features are less present for the STM images measured at bias voltages of 1 and $2 \mathrm{~V}$.

Figure $3 \mathrm{e}$ presents the line profiles corresponding to the STM images illustrated in Figure $3 a-d$. BNTCDI shows the highest corrugation at $-1.5 \mathrm{~V}$ with a relative low electronic density on the phenyl groups and the center of the molecule. The absolute values of the apparent height of the blue $(+0.25$ $\mathrm{V})$, red $(+1.0 \mathrm{~V})$, and orange $(+2.0 \mathrm{~V})$ line profiles are larger than those of the black curve $(-1.5 \mathrm{~V})$ at the site of the NDI core and the phenyl groups. Consequently, a lower apparent height difference between the two bright spots (above nitrogen atoms) and the center of the molecule results. As the apparent height profiles indicate, these STM images show less distinguishable features with increasing voltage.

Identification of Molecular-Like Orbitals. In order to unravel the mechanism of the submolecular resolution observed in the STM images that suggest a weak molecule-substrate interaction, ${ }^{20,25}$ we performed first principle simulations for the BNTCDI (i) in the gas phase and (ii) in the STM configuration. More precisely, in a first step, we investigated the BNI molecule in the gas phase considering several molecular conformers using density functional theory (DFT). For the relaxed geometry reported in Figure 2a,b, the distance between the center of the phenyl rings is similar to the one measured in the STM images shown in Figures $2 \mathrm{c}$ and 3.

By comparing the voltage dependent STM images (see Figure 3) with the charge density distribution of the last two occupied (HOMO-1, HOMO) and first two unoccupied (LUMO, LUMO+1) frontier molecular orbitals (Figure 4) of the BNTCDI molecule in the gas phase, we can identify specific common features for the NDI core and the phenyl rings. More precisely, while at the NDI core the nodal planes are always between different atoms, the top view images of the $\pi$-like orbitals located at the phenyl rings (Figure 4a,d,g) reveal the presence of an atomic driven nodal plane for both occupied and unoccupied molecular orbitals. This nodal plane separates the lobes of the $\mathrm{p}_{z}$-atomic-like orbitals that form the $\pi$-orbitals located at the phenyl group. Such a nodal plane is clearly observed in our STM measurements, as shown in Figures $2 \mathrm{c}$ and $3 \mathrm{~b}$. This represents the visualization of an in-plane $\pi$-nodal plane present at the $\mathrm{C}$ atomic positions of an aromatic ring 
within a STM experiment. To the best of our knowledge, this has been reported up to now only for a small molecule, nitrosobenzene, chemisorbed on $\mathrm{Ge}(100) .{ }^{51}$ Note that, in the case of flat two-dimensional molecules such as pentacene, ${ }^{20}$ the STM measurements revealed the presence of out-of-plane nodal planes between different carbon atoms.

The side views of the charge density plots corresponding to the calculated molecular orbitals shown in Figure 4c,f,i,l indicate that close to the BNTCDI molecule the charge density isolines exhibit a corrugation similar to that of the line profiles reported in Figure 3e. In particular, the corrugation depicted in these side views is more pronounced for HOMO-1 and HOMO (see Figure 4c,f) and smaller for LUMO and LUMO+1 (Figure 4i,1). This is in qualitative agreement with the experimental apparent height profiles (see Figure 3e) showing a higher corrugation for the occupied electronic states measured at negative bias voltages as compared to unoccupied states measured at positive bias voltages.

Transport Properties of BNTCDI. In a second step, we investigated the transport properties of the physisorbed BNTCDI molecule on $\mathrm{Pt}(111)$. We considered a moleculesurface system within a simple model; i.e., the molecule is adsorbed at $0.3 \mathrm{~nm}$ above a jellium electrode, while the STM tip is modeled as a $\mathrm{W}$ apex atom attached to the upper jellium electrode at a distance of $0.1 \mathrm{~nm}$. We evaluated the electron tunneling transport for two distances between the tip and the highest $\mathrm{H}$ atom of the molecule $(0.5$ and $0.8 \mathrm{~nm})$ and four tip positions, as shown in the inset of Figure 5 (i.e., the tip atom is located above the molecular center, the nitrogen atom, and the two topmost hydrogen atoms of the BNTCDI molecule).

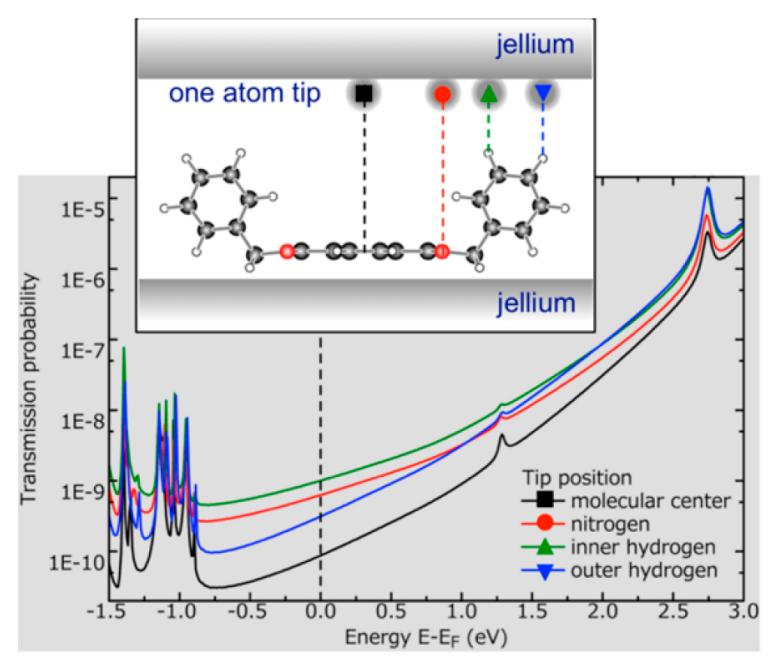

Figure 5. Electron transmission spectra for a tip-molecule distance of $0.8 \mathrm{~nm}$ with respect to the topmost hydrogen atom of the phenyl ring. Inset: Cartoon of the geometry used in the simulations.

As can be seen from Figure 5, even at zero bias $\left(E_{\mathrm{F}}\right)$, the calculated transmission probability varies significantly for different tip positions above the molecule. More precisely, the conductance is much lower when the tip is above the NDI core with respect to that obtained above phenyl rings. This observation clearly emphasizes that the 3D structure of the molecules is reflected in the constant current STM images. This implies that even around the Fermi energy the STM tip will approach the NDI core and will be withdrawn above phenyl rings. In addition, for the tip position above the NDI plane and above the highest $\mathrm{H}$ atom of the phenyl arm, the difference in transmission probabilities is larger for negative bias values than for positive ones. This again is in a qualitative agreement with the experimental apparent height profiles (Figure $3 \mathrm{e}$ ) and the corrugation of charge density isolines plotted in Figure $4 \mathrm{c}, \mathrm{f}, \mathrm{i}, \mathrm{l}$.

Furthermore, we performed a scanning tunneling spectroscopy (STS) analysis and acquired conductivity curves above the center and the periphery of BNTCDI adsorbed on $\mathrm{Pt}(111)$ (see Figure 6). However, they do not show a distinct HOMO-

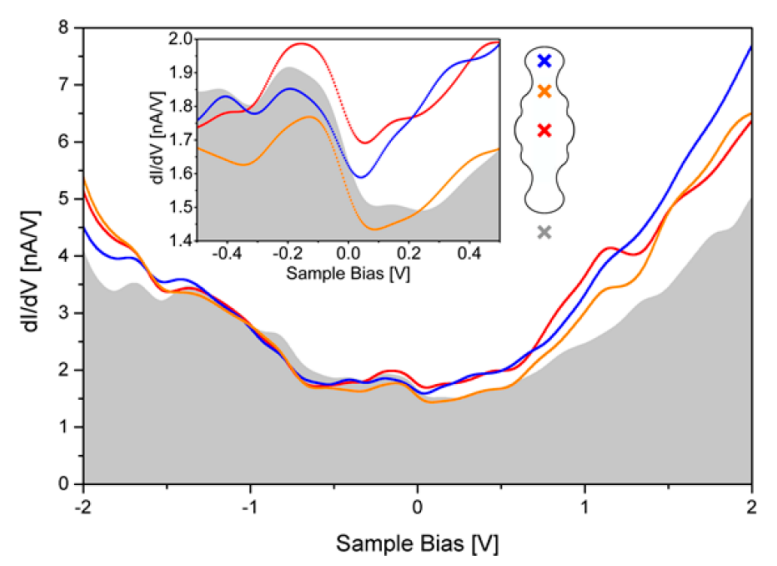

Figure 6. STS measurements on the $\mathrm{Pt}(111)$ substrate (gray background), the center of BNTCDI (red), the bright spots (orange), and the outer part of BNTCDI (blue).

LUMO gap (HLG) as in the case of physisorbed $\pi$-molecular systems. ${ }^{20}$ More specifically, around the Fermi energy, the conductivity spectra taken above the BNTCDI molecule have a magnitude similar to that of the metallic $\mathrm{Pt}(111)$ substrate. The best compliance is found between the $d I / d V$ curve taken above a bright spot, located near the $\mathrm{N}$ atoms of BNTCDI (orange curve in Figure 6), and the one above the $\mathrm{Pt}(111)$ substrate, indicating a locally increased interaction. This behavior indicates that BNTCDI is not physisorbed but rather weakly chemisorbed on $\operatorname{Pt}(111)$.

It has been observed recently that the introduction of functional groups with acceptor character into planar aromatic hydrocarbons facilitates the hybridization of the molecular orbitals with metallic surface states. ${ }^{32,34}$ Thus, an increased coupling of these molecules to the substrate compared to unsubstituted perylenes results. Accordingly, the adsorption of NDI on $\mathrm{Si}(111)$ induces significant charge redistribution in order to form a chemical bond with the surface. ${ }^{31}$ As a consequence, the LUMO states shift toward lower energies upon adsorption, as reported for PTCDA and TCNQ, belonging to the class of functionalized planar aromatic hydrocarbons with acceptor character. ${ }^{35}$ The extent of the shift depends on the interaction strength with the respective substrate.

In the case of BNTCDI adsorbed on $\mathrm{Pt}(111)$, we find that the STM images obtained around the Fermi energy for occupied as well as unoccupied molecule-surface electronic states exhibit specific features that are characteristic for the LUMO of BNTCDI in the gas phase (see Figures 4g,h and 7). In detail, these are the two regions of decreased charge density marked by dashed blue lines at the NDI core, the four spots showing the $\mathrm{p}_{z}$-like orbitals forming the $\pi$-orbitals at the phenyl rings (emphasized by light blue ellipses), and the nodal plane in between these features. Due to the molecular 3D structure, the 
(a)

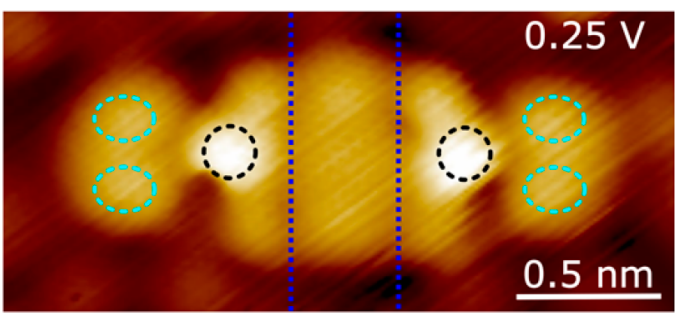

(b)

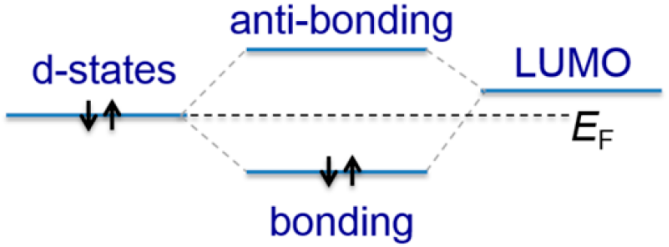

(c)

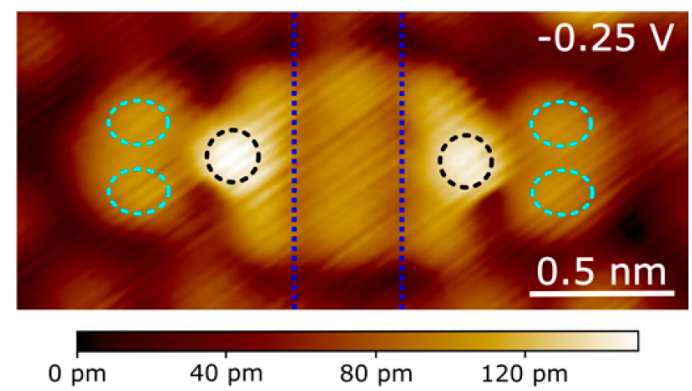

Figure 7. Schematic depiction of the interaction between the Pt $d$ states and the LUMO of the BNTCDI molecule (b). STM images obtained for a bias voltage of $+0.25 \mathrm{~V}$ (a) and $-0.25 \mathrm{~V}$ (c). Light blue markers emphasize the $\pi$-orbitals at the phenyl rings, black circles correspond to charge densities above $\mathrm{N}$ atoms, and the blue dashed lines show the regions of decreased charge density at the NDI core.

STM image acquired above the phenyl rings resembles the LUMO features shown in Figure 4g,i,e, at a height of $1 \mathrm{~nm}$ above the NDI core, while the STM image obtained on top of the NDI core is similar to the LUMO features shown in Figure $4 \mathrm{~h}$, i.e., at a height of $0.2 \mathrm{~nm}$ above the NDI core. The observation of LUMO features near the Fermi energy confirms the interaction between the $\mathrm{Pt} d$-states and the LUMO of BNTCDI that leads to bonding and antibonding states of the molecule-surface system. As a consequence, the top views of both bonding and antibonding molecule-surface states will have a similar appearance, as indeed experimentally observed in Figure $7 \mathrm{a}$ and $\mathrm{c}^{17}$

Besides this, as already mentioned, all STM experimental images indicate the presence of two bright spots (see black dashed marked regions in Figure $7 \mathrm{a}, \mathrm{c}$ ) at approximately 0.86 $\mathrm{nm}$ apart from each other. These two features are not present in the molecular orbital pictures obtained for BNTCDI in the gas phase, i.e., assuming that it is physisorbed on the $\mathrm{Pt}(111)$ substrate. They can be located near the $\mathrm{N}$ atoms within the molecule according to geometrical analysis. One reason for the increased charge density at these sites is the charge redistribution due to chemical interactions of the functional molecular groups with the substrate discussed above. Another point is the charge density associated with the two hydrogen bonds formed between $\mathrm{N}$ atoms and the neighboring $\mathrm{H}$ atoms of the phenyl ring (see Figure $2 b$ ) also located at the site of the $\mathrm{N}$ atoms (top view). Therefore, we assume that these bright spots originate from weak local $\mathrm{Pt} \cdots \mathrm{N} \cdots \mathrm{H}-\mathrm{C}$ chemical interactions $^{52}$ that lead to an opening of tunneling channels around the Fermi energy. They represent the most efficient transport paths, like also suggested by the electron transmission spectra (Figure 5), for electrons tunneling from the tip to the $\mathrm{Pt}(111)$ substrate through the BNTCDI molecule and vice versa.

\section{CONCLUSION}

To summarize, in this combined experimental and theoretical study, we investigated the geometrical and electronic structure of a 3D molecule such as 2,7-dibenzyl 1,4,5,8-naphthalenetetracarboxylic diimide (BNTCDI) weakly chemisorbed on Pt(111). Importantly, the STM experiments performed for this system revealed a submolecular resolution for BNTCDI corresponding to $\pi$-conjugated systems oriented perpendicular to each other. This implies that we visualize via STM an inplane $\pi$-nodal plane (i.e., that separates the lobes of the $\mathrm{p}_{z^{-}}$ atomic-like orbitals) present at the $\mathrm{C}$ atomic positions of the phenyl rings. This is a challenging achievement noticing that in such STM experiments the current signal varies significantly due to the height differences within a $3 \mathrm{D}$ molecule, leading overall to a decreased resolution. Indeed, in our first principle calculations, we demonstrated that the convolution between the 3D shape of BNTCDI and its electronic structure plays a crucial role in the STM imaging process. Thereby, the presentation of the charge density plots at different heights above the planar backbone of the molecule has been proved to be a useful tool to identify STM features resulting from molecular-like orbitals measured in constant current mode.

In particular, our STM measurements in combination with DFT calculations indicate the presence of hybrid BNTCDI/Pt $d$-states around the Fermi energy and local $\mathrm{N} \cdots \mathrm{H}-\mathrm{C}$ hydrogen bonds that open an effective transport path around the Fermi energy. Hence, we expect that such a methodology based on STM and DFT can be successfully applied for other adsorbed $\pi$-conjugated molecules whose $3 \mathrm{D}$ geometrical shapes lead to large height differences due to protruding molecular groups.

\section{ASSOCIATED CONTENT}

\section{Supporting Information}

The Supporting Information is available free of charge on the ACS Publications website at DOI: 10.1021/acs.jpcc.7b09911.

Thermogravimetric (TG), differential thermal (DT) analysis, UV/vis absorption spectrum, and cyclic voltammetry of BNTCDI, LT-UHV-STM images, and apparent height profiles (PDF)

\section{AUTHOR INFORMATION}

\section{Corresponding Authors}

*E-mail: s.karthaeuser@fz-juelich.de. Phone: +49 2461614015. *E-mail: n.atodiresei@fz-juelich.de. Phone: +49 2461614745.

\section{ORCID}

S. Tsukamoto: 0000-0001-5271-9277

S. Karthäuser: 0000-0003-3953-6980

\section{Author Contributions}

The manuscript was written through contributions of all authors. All authors have given approval to the final version of the manuscript.

\section{Notes}

The authors declare no competing financial interest. 


\section{ACKNOWLEDGMENTS}

We cordially thank Katarzyna Skierkowska and Prof. Dr. Walter Frank for thermogravimetric and differential thermal analyses. Computations were carried out using the high-performance computer JUQUEEN and JURECA operated by the Jülich Supercomputing Centre at the Forschungszentrum Jülich $\mathrm{GmbH}$. The authors gratefully acknowledge financial support from the Volkswagen Stiftung through the "Optically Controlled Spin Logic" project and from the DFG through the CRC1238 (project C01).

\section{REFERENCES}

(1) Suraru, S.-L.; Würthner, F. Strategies for the Synthesis of Functional Naphthalene Diimides. Angew. Chem., Int. Ed. 2014, 53, 7428-7448.

(2) Bhosale, S. V.; Jani, C. H.; Langford, S. J. Chemistry of Naphthalene Diimides. Chem. Soc. Rev. 2008, 37, 331-342.

(3) Chen, W.; Zhang, Q. Recent Progress in Non-Fullerene Small Molecule Acceptors in Organic Solar Cells (OSCs). J. Mater. Chem. C 2017, 5, 1275-1302.

(4) Benten, H.; Mori, D.; Ohkita, H.; Ito, S. Recent Research Progress of Polymer Donor/Polymer Acceptor Blend Solar Cells. J. Mater. Chem. A 2016, 4, 5340-5365.

(5) Zhan, C.; Zhang, X.; Yao, J. New Advances in Non-Fullerene Acceptor Based Organic Solar Cells. RSC Adv. 2015, 5, 93002-93026.

(6) Lin, Y.; Zhan, X. Non-Fullerene Acceptors for Organic

Photovoltaics: An Emerging Horizon. Mater. Horiz. 2014, 1, 470-488.

(7) Jiang, W.; Li, Y.; Wang, Z. Tailor-Made Rylene Arrays for High Performance n-Channel Semiconductors. Acc. Chem. Res. 2014, 47, 3135-3147.

(8) Zhao, Y.; Guo, Y.; Liu, Y. 25th Anniversary Article: Recent Advances in n-Type and Ambipolar Organic Field-Effect Transistors. Adv. Mater. 2013, 25, 5372-5391.

(9) Zhan, X.; Facchetti, A.; Barlow, S.; Marks, T. J.; Ratner, M. A.; Wasielewski, M. R; Marder, S. R. Rylene and Related Diimides for Organic Electronics. Adv. Mater. 2011, 23, 268-284.

(10) Sugimura, R.; Suzuki, S.; Kozaki, M.; Keyaki, K.; Nozaki, K.; Matsushita, H.; Ikeda, N.; Okada, K. Photoinduced Charge Separation of Phenothiazine-Platinum-Naphthalene Diimide Triads Linked by Twisted Phenylene Bridges. Res. Chem. Intermed. 2013, 39, 185-204.

(11) Pan, M.; Lin, X.-M.; Li, G.-B.; Su, C.-Y. Progress in the Study of Metal-Organic Materials Applying Naphthalene Diimide (NDI) Ligands. Coord. Chem. Rev. 2011, 255, 1921-1936.

(12) Langford, S. J.; Latter, M. J.; Woodward, C. P. Progress in Charge Transfer Systems Utilizing Porphyrin Donors and Simple Aromatic Diimide Acceptor Units. Photochem. Photobiol. 2006, 82, $1530-1540$.

(13) Ju, H. X.; Zhao, H. T. Electrochemical Biosensors for DNA Analysis. Front. Biosci. (Landmark Ed) 2005, 10, 37-46.

(14) Doria, F.; Folini, M.; Grande, V.; Cimino-Reale, G.; Zaffaroni, N.; Freccero, M. Naphthalene Diimides as Red Fluorescent $\mathrm{pH}$ Sensors for Functional Cell Imaging. Org. Biomol. Chem. 2015, 13, 570-576.

(15) Thalacker, C.; Röger, C.; Würthner, F. Synthesis and Optical and Redox Properties of Core-Substituted Naphthalene Diimide Dyes. J. Org. Chem. 2006, 71, 8098-8105.

(16) Lennartz, M. C.; Caciuc, V.; Atodiresei, N.; Karthäuser, S.; Blügel, S. Electronic Mapping of Molecular Orbitals at the MoleculeMetal Interface. Phys. Rev. Lett. 2010, 105, 066801.

(17) Atodiresei, N.; Brede, J.; Lazic, P.; Caciuc, V.; Hoffmann, G.; Wiesendanger, R.; Blügel, S. Design of the Local Spin Polarization at the Organic-Ferromagnetic Interface. Phys. Rev. Lett. 2010, 105, 066601 .

(18) Harutyunyan, H.; Callsen, M.; Allmers, T.; Caciuc, V.; Blügel, S.; Atodiresei, N.; Wegner, D. Hybridization at the Organic-Metal Interface: A Surface-Scientific Analogue of Huckel's Rule? Chem. Commun. 2013, 49, 5993.
(19) Brede, J.; Atodiresei, N.; Kuck, S.; Lazic, P.; Caciuc, V.; Morikawa, Y.; Hoffmann, G.; Blügel, S.; Wiesendanger, R. Spin- and Energy-Dependent Tunneling through a Single Molecule with Intramolecular Spatial Resolution. Phys. Rev. Lett. 2010, 105, 047204.

(20) Repp, J.; Meyer, G.; Stojkovic, S.; Gourdon, A.; Joachim, C. Molecules on Insulating Films: Scanning-Tunneling Microscopy Imaging of Individual Molecular Orbitals. Phys. Rev. Lett. 2005, 94, 026803.

(21) Martinez-Galera, A.; Nicoara, N.; Martinez, J.; Dappe, Y.; Ortega, J.; Gomez-Rodriguez, J. Imaging Molecular Orbitals of PTCDA on Graphene on $\operatorname{Pt}(111)$ : Electronic Structure by STM and First-Principles Calculations. J. Phys. Chem. C 2014, 118, 1278212788 .

(22) Kumar, A.; Banerjee, K.; Liljeroth, P. Molecular Assembly on Two-Dimensional Materials. Nanotechnology 2017, 28, 082001.

(23) Paßens, M.; Moors, M.; Waser, R.; Karthäuser, S. Charge Transport across the $\mathrm{C}_{60} / \mathrm{TiO}$ Ultrathin Film Interface. J. Phys. Chem. C 2017, 121, 2815-2821.

(24) Garnica, M.; Stradi, D.; Barja, S.; Calleja, F.; Diaz, C.; Alcami, M.; Martin, N.; de Parga, A. L. V.; Martin, F.; Miranda, R. Long-Range Magnetic Order in a Purely Organic 2D Layer Adsorbed on Epitaxial Graphene. Nat. Phys. 2013, 9, 368-374.

(25) Neél, N.; Lattelais, M.; Bocquet, M.-L.; Kröger, J. Depopulation of Single Phthalocyanine Molecular Orbitals upon Pyrrolic-Hydrogen Abstraction on Graphene. ACS Nano 2016, 10, 2010-2016.

(26) Hollerer, M.; Lüftner, D.; Hurdax, P.; Ules, T.; Soubatch, S.; Tautz, F. S.; Koller, G.; Puschnig, P.; Sterrer, M.; Ramsey, M. G. Charge Transfer and Orbital Level Alignment at Inorganic/Organic Interfaces: The role of Dielectric Interlayers. ACS Nano 2017, 11, 6252-6260.

(27) De Oteyza, D.; Gorman, P.; Chen, Y.-C.; Wickenburg, S.; Riss, A.; Mowbray, D.; Ethin, G.; Pedramrazi, Z.; Tsai, H. Z.; Rubio, A.; et al. Direct Imaging of Covalent Bond Structure in Single-Molecule Chemical Reactions. Science 2013, 340, 1434-1437.

(28) Weiss, C.; Wagner, C.; Kleimann, C.; Rohlfing, M.; Tautz, F. S.; Temirov, R. Imaging Pauli Repulsion in Scanning Tunneling Microscopy. Phys. Rev. Lett. 2010, 105, 086103.

(29) Gross, L.; Mohn, F.; Moll, N.; Liljeroth, P.; Meyer, G. The Chemical Structure of a Molecule Resolved by Atomic Force Microscopy. Science 2009, 325, 1110-1114.

(30) Sweetman, A.; Jarvis, S.; Sang, H.; Lekkas, I.; Rahe, P.; Wang, Y.; Wang, J.; Champness, N.; Kantorovich, L.; Moriarty, P. Mapping the Force Field of a Hydrogen-Bonded Assembly. Nat. Nat. Commun. 2014, 5, 3931.

(31) Jarvis, S.; Sweetman, A.; Lekkas, I.; Champness, N.; Kantorovich, L.; Moriarty, P. Simulated Structure and Imaging of NTCDI on $\mathrm{Si}(111)-7 \times 7$ : A Combined STM, NC-AFM and DFT study. J. Phys.: Condens. Matter 2015, 27, 054004.

(32) Mistry, A.; Moreton, B.; Schuler, B.; Mohn, F.; Meyer, G.; Gross, L.; Williams, A.; Scott, P.; Costantini, G.; Fox, D. The Synthesis and STM/AFM Imaging of Olympicene Benz $[\mathrm{dc}]$ pyrenes. Chem. Eur. J. 2015, 21, 2011-2018.

(33) Kowalzik, P.; Atodiresei, N.; Gingras, M.; Caciuc, V.; Blügel, S.; Waser, R.; Karthäuser, S. Single Electron Tunneling through a Tailored Arylthio-Coronene. J. Phys. Chem. C 2011, 115, 9204-9209.

(34) Franco-Canellas, A.; Wang, Q.; Broch, K.; Duncan, D. A.; Thakur, P. K.; Liu, L.; Kera, S.; Gerlach, A.; Duhm, S.; Schreiber, F. Metal-Organic Interface Functionalization via Acceptor End Groups: PTCDI on Coinage Metals. Phys. Rev. Mater. 2017, 1, No. 013001(R), DOI: 10.1103/PhysRevMaterials.1.013001.

(35) Otero, R.; Vázquez de Parga, A. L.; Gallego, J. M. Electronic, Structural and Chemical Effects of Charge-Transfer at Organic/ Inorganic Interfaces. Surf. Sci. Rep. 2017, 72, 105-145.

(36) Cao, D.; Amelia, M.; Klivansky, L. M.; Koshkakaryan, G.; Khan, S. I.; Semeraro, M.; Silvi, S.; Venturi, M.; Credi, A.; Liu, Y. Probing Donor-Acceptor Interactions and $\mathrm{Co}$-Conformational Changes in Redox Active Desymmetrized [2]Catenanes. J. Am. Chem. Soc. 2010, $132,1110-1122$. 
(37) Tsukamoto, S.; Egami, Y.; Hirose, K.; Blügel, S. Stabilized Scattering Wave-Function Calculations Using the LippmannSchwinger Equation for Long Conductor Systems. Phys. Rev. B: Condens. Matter Mater. Phys. 2011, 84, 115443.

(38) Lang, N. D. Resistance of Atomic Wires. Phys. Rev. B: Condens. Matter Mater. Phys. 1995, 52, 5335-5342.

(39) Blöchl, P. E. Projector Augmented-Wave Method. Phys. Rev. B: Condens. Matter Mater. Phys. 1994, 50, 17953-17979.

(40) Vosko, S. H.; Wilk, L.; Nusair, M. Accurate Spin-Dependent Electron Liquid Correlation Energies for Local Spin Density Calculations: A Critical Analysis. Can. J. Phys. 1980, 58, 1200-1211.

(41) Büttiker, M.; Imry, Y.; Landauer, R.; Pinhas, S. Generalized Many-Channel Conductance Formula with Application to Small Rings. Phys. Rev. B: Condens. Matter Mater. Phys. 1985, 31, 6207-6215.

(42) Seydou, M.; Teyssandier, J.; Battaglini, N.; Kenfack, G. T.; Lang, P.; Tielens, F.; Maurel, F.; Diawara, B. Characterization of NTCDI Supra-Molecular Networks on $\mathrm{Au}(111)$; Combining STM, IR and DFT Calculations. RSC Adv. 2014, 4, 25698-25708.

(43) Fu, C.; Lin, H.-P.; Macleod, J. M.; Krayev, A.; Rosei, F.; Perepichka, D. F. Unravelling the Self-Assembly of Hydrogen Bonded NDI Semiconductors in 2D and 3D. Chem. Mater. 2016, 28, 951-961.

(44) Teyssandier, J.; Battaglini, N.; Seydou, M.; Anquetin, G.; Diawara, B.; Sun, X.; Maurel, P.; Lang, P. Elaboration of HydrogenBonded 2D Supramolecular Assemblies on $\mathrm{Au}(111)$ from Solutions: Toward Naphthalene Tetracarboxylic Diimide - Melamine Nanoporous Networks. J. Phys. Chem. C 2013, 117, 8737-8745.

(45) Kakinuma, T.; Kojima, H.; Ashizawa, M.; Matsumoto, H.; Mori, T. Correlation of Mobility and Molecular Packing in Organic Transistors Based on Cycloalkyl Naphthalene Diimides. J. Mater. Chem. C 2013, 1, 5395-5401.

(46) Miyake, Y.; Nagata, T.; Tanaka, H.; Yamazaki, M.; Ohta, M.; Kokawa, R.; Ogawa, T. ACS Nano 2012, 6, 3876-3887.

(47) Seebach, D.; Groselj, U.; Schweizer, W. B.; Grimme, S.; MückLichtenfeld, C. Experimental and Theoretical Conformation Analysis of 5-Benzylimidazolidin-4-One Derivatives - a Playground for Studying Dispersion Interactions and a Windshield-Wiper Effect in Organocatalysis. Helv. Chim. Acta 2010, 93, 1-16.

(48) Zhou, C.; Deshpande, M. R.; Reed, M. A.; Jones, L., II; Tour, J. M. Nanoscale Metal/Self-Assembled Monolayer/Metal Heterostructures. Appl. Phys. Lett. 1997, 71, 611-613.

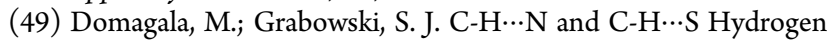
Bonds - Influence of Hybridization on their Strength. J. Phys. Chem. A 2005, 109, 5683-5688.

(50) Spada, L.; Gou, Q.; Vallejo-López, M.; Lesarri, A.; Cocinero, E. J.; Caminati, W. Weak C-H $\cdots \mathrm{N}$ and $\mathrm{C}-\mathrm{H} \cdots \mathrm{F}$ Hydrogen Bonds and Internal Rotation in Pyridine- $\mathrm{CH}_{3}$ F. Phys. Chem. Chem. Phys. 2014, 16, 2149-2153.

(51) He, J. H.; Mao, W.; Chen, W.; Wu, K.; Cheng, H. S.; Xu, G. Q. Resolving Molecular Orbitals Self-Decoupled from Semiconductor Surfaces. Chem. Sci. 2014, 5, 4447-4452.

(52) Ahmadi, S.; Agnarsson, B.; Bidermane, I.; Wojek, B. M.; Noel, Q.; Sun, C. H.; Göthelid, M. Site-Dependent Charge Transfer at the $\mathrm{Pt}(111)-\mathrm{ZnPc}$ Interface and the Effect of Iodine. J. Chem. Phys. 2014, 140, 174702. 